\title{
REVISÃO SISTEMÁTICA DA PRODUÇÃO CIENTÍFICA RELACIONADA À ATIVIDADE FÍSICA E QUALIDADE DE VIDA DE MILITARES
}

\author{
Systematic revision of the scientific production related to the physical activity and \\ quality of life of military individuals
}

\author{
Antônio Melo Schlichting Junior ${ }^{1}$, Rudney da Silva ${ }^{1}$ \\ ${ }^{1}$ Laboratório de Atividade Motora Adaptada - Centro de Ciências da Saúde e do Esporte \\ Universidade do Estado de Santa Catarina - Florianópolis - SC - Brasil
}

\begin{abstract}
Resumo: As sociedades modernas vêm sofrendo mudanças que afetam diretamente os indivíduos. Os avanços científicos e tecnológicos têm apresentado interferências na saúde e na qualidade de vida daqueles expostos ao mundo do trabalho. Assim, pode-se sugerir que o trabalho do militar configura-se como uma temática que merece atenção científica, principalmente pelas exigências físicas e mentais destes indivíduos no exercício de suas funções, essenciais à sociedade. Diante do exposto, este estudo tem por objetivo desenvolver uma revisão sistemática sobre a produção científica brasileira no período de janeiro de 2003 a março de 2008, com ênfase em aspectos relacionados à atividade física e qualidade de vida de militares. Os procedimentos incluíram a seleção das bases de dados e a identificação de produções científicas através de buscas simples e booleana. As bases de dados selecionadas foram Scielo e Google. Depois de selecionadas as bases de dados, foi pesquisado o descritor primário "militar" identificandose 16.900 citações. Na busca booleana realizou-se o cruzamento do descritor primário com os descritores secundários "nível de atividade física" e "qualidade de vida", identificando-se 120 estudos. Após as análises preliminares, baseadas em critérios de seleção, foram identificados 28 estudos. 0 estudo crítico da metodologia adotada e dos resultados obtidos permitiu identificar 17 artigos agrupados em dois estratos básicos: a) Militares Federais (11 artigos); b) Militares Estaduais (seis estudos). Assim, pode-se concluir que ocorre maior número de estudos com militares federais, com maior ênfase no treinamento militar e menor ênfase na aptidão física relacionada à saúde. Já os estudos com militares estaduais apresentam maior ênfase na aptidão física relacionada à saúde e menor ênfase em temáticas voltadas à atividade profissional, à capacidade de trabalho e ao estilo de vida.
\end{abstract}

Palavras-chave: Nível de Atividade Física, Qualidade de Vida, Militares.

\begin{abstract}
The modern societies are suffering changes that affect the individuals directly. The scientific and technological progresses to have interferences in the health and in the quality of life individuals exposed to the world of the work. Thus, it can be suggested that the work of the military individuals is configured as a thematic that to need scientific attention, principally for the physical and mental demands in the exercise of its functions, essential to the society. As displayed, this study has for objective to develop a systematic revision about the Brazilian scientific production in the period of january of 2003 to march of 2008 , with emphasis in aspects related to the physical activity and life quality of military individuals. The procedures included the selection of the databases and the identification of scientific productions through simple and Boolean searches. They were selected the databases Scielo and Google. After the selected databases, it was researched the primary descriptor "military individuals" identifying 16.900 citations. In the search Boolean the crossing of the primary descriptor with the secondary descriptors "level of physical activity" and "quality of life", identifying 120 studies. After the preliminary analyses, based on the selection through approaches, it was identified 28 studies. The critical study it analyzed of the adopted methodology and of the obtained results, identifying 17 studies in two basic groups: a) Federal Military individuals (11 studies); b) State Military Individuals (six studies). Thus, it can be concluded that happens bigger number of studies with federal military individuals, with larger emphasis in the military training and smaller emphasis in the physical aptitude related to the health. Already the studies with state military individuals, it was verified bigger emphasis in the physical aptitude related to the health and smaller emphasis in thematic related to the professional activity, to the work capacity and the lifestyle.
\end{abstract}

Key words: Level of Physical Activity, Quality of Life, Military Individuals.

Aceito em 20/02/2009 - Rev. Educ. Fís. 2009 Jun: 145: 28-36. Rio de Janeiro - RJ - Brasil

\section{INTRODUÇÃO}

As sociedades modernas vêm gradativamente sofrendo mudanças que afetam os indivíduos sob diferentes formas. Os avanços científicos e tecnológicos que facilitam a vida humana, provocam um paradoxo que interfere diretamente na saúde e na qualidade de vida dos indivíduos, pois concomitantemente ao conforto inerente à diminuição das exigências físicas, vêm causando diminuição no nível de exercícios físicos ${ }^{(1)}$.

O exercício físico efetuado de forma contínua e 
constante, ou seja, fazendo parte do cotidiano dos indivíduos, é considerado como um dos fatores com maior impacto não só na prevenção de enfermidades, tais como obesidade, diabetes, cardiopatias e depressão, mas também na manutenção e autonomia funcional do sujeito, tanto no lazer quanto no trabalho(2).

O trabalho humano sofre interferência direta dos modelos social, político e econômico que derivaram da Revolução Industrial e afeta os indivíduos nos seus aspectos fisiológico, psicológico e sociológico ${ }^{(3,4)}$. Dentre as variadas possibilidades de trabalho na atualidade, existem profissões que causam maior desgaste cognitivo e outras desgastes físicos ${ }^{(5,6)}$.

As profissões que exigem maior esforço cognitivo, provocam cargas psíquicas intensas, causando psicopatologias variadas ${ }^{(6)}$. Já as profissões que exigem maior esforço físico, causam danos principalmente aos sistemas cardiovascular e ostemioarticular ${ }^{(5)}$. Entretanto, tanto as enfermidades psíquicas quanto físicas provocam diminuição da qualidade de vida dos indivíduos ${ }^{(5,6)}$.

De forma duplamente afetada, existem profissões que exigem tanto o esforço físico acentuado quanto impõem intensa pressão psíquica no desenvolvimento de suas funções, como por exemplo, os profissionais militares. No Brasil, os militares podem ser caracterizados pelas forças armadas e pelas auxiliares ${ }^{(7,8)}$. As Forças Armadas são constituídas pela Marinha, Exército e Aeronáutica e são denominados militares federais e objetivam a defesa da pátria ${ }^{(7)}$. Já os Policias Militares e Bombeiros Militares, denominados militares estaduais, são considerados forças auxiliares e reservas e tem como missão a policia ostensiva e preservação da ordem pública ${ }^{(8)}$. Assim, considerando os diferentes contextos sociais atuais, pode-se afirmar que a segurança pública configura-se como um grave problema social, e que os profissionais que nela atuam merecem atenção científica adequada ${ }^{(9)}$.

Diante deste contexto, este estudo teve como objetivo desenvolver uma revisão sistemática sobre a produção científica brasileira no período de janeiro de 2003 a março de 2008, com ênfase em aspectos relacionados à atividade física e qualidade de vida de militares.

\section{METODOLOGIA}

Esta pesquisa, caracterizada como um estudo bibliográfico e documental(10,11), buscou revisar a produção científica brasileira disponível em bases de dados de acesso público e no idioma português, no período de janeiro de 2003 a março de 2008, segundo o quadro operacional construído para este estudo, conforme FIGURA 1.

\section{FIGURA 1}

\section{QUADRO OPERACIONAL DA PESQUISA}

\begin{tabular}{ccc}
\hline CATEGORIA & SUBCATEGORIAS & TEMAS \\
\hline & Aptidão \\
& Física; Força, \\
& Flexibilidade, \\
& Composição \\
& Atividade Física & Corporal; \\
& Resistência \\
& Aeróbica; \\
Militar & Resistência \\
& & Anaeróbica. \\
\cline { 2 - 3 } & & Trabalho; \\
& & Estresse; \\
& & Qualidade de Vida \\
& Saúde; Estilo de \\
& Vida. \\
\hline
\end{tabular}

A elaboração de um quadro operacional, mostrando os indicadores do estudo, possibilita uma materialização daquilo que se pretende observar, pois permite a passagem da linguagem abstrata do conceito para a linguagem concreta da observação empírica, possibilitando assim análise da realidade e a interpretação dos fundamentos teóricos do trabalho(11).

Para tanto, foram analisados artigos científicos, monografias de graduação e pós-graduação, dissertações e teses, disponíveis na rede mundial de computadores - Internet. $\mathrm{O}$ desenvolvimento 
do estudo ocorreu através de duas etapas para o processo de revisão sistemática: 1) Identificação e seleção das bases dados; 2) Levantamento e análise da produção científica brasileira.

A primeira etapa visou à seleção de bases de dados que atendessem a três critérios de inclusão: 1) ser caracterizada como uma base de dados cientificamente confiável; 2) disponibilizar gratuita e integralmente os materiais eletrônicos; 3 ) ser apresentada em língua portuguesa. Deste modo, foi possível selecionar duas bases de dados em seus sítios eletrônicos na rede mundial de computadores: a) Google Acadêmico; b) Scielo.

O Google Acadêmico configura-se como um recurso de busca, que possibilita pesquisas na literatura acadêmica disponível na rede mundial de computadores, permitindo levantar artigos revisados por especialistas, teses, livros, resumos e artigos de editoras acadêmicas, organizações profissionais, bibliotecas de pré-publicações, universidades e outras entidades acadêmicas, assim como identificar as pesquisas com maior relevância acadêmica.

A Scielo é uma biblioteca eletrônica que abrange uma coleção selecionada de periódicos científicos brasileiros e que resulta da associação entre a Fundação de Amparo à Pesquisa do Estado de São Paulo e Centro Latino-Americano e do Caribe de Informação em Ciências da Saúde. A Scielo objetiva o desenvolvimento de uma metodologia comum para a preparação, armazenamento, disseminação e avaliação da produção científica em formato eletrônico, proporcionando amplo acesso a coleções de periódicos como um todo, aos fascículos de cada título de periódico, assim como aos textos completos dos artigos.

A segunda etapa objetivou o levantamento e análise de produções científicas brasileiras, e foi desenvolvida através de quatro passos: 1) busca simples de produções científicas sobre a categoria principal deste estudo, o descritor "militar". Este procedimento permitiu a identificação de cerca de 16.900 citações; 2) cruzamento da categoria principal com as subcategorias do estudo (busca boleana), neste caso, os descritores "nível de atividade física" e "qualidade de vida". Este procedimento permitiu a identificação de 120 trabalhos; 3) análise preliminar de estudos que atendessem basicamente a dois critérios de análise: a) relevância do artigo aos termos investigados; b) descritores relacionados aos termos investigados. Este procedimento permitiu identificar 28 estudos; 4) estudo minucioso dos artigos analisando-se criticamente a metodologia adotada e os resultados obtidos. Este procedimento permitiu identificar 17 artigos, os quais foram agrupados em dois estratos básicos: a) Militares Federais; b) Militares Estaduais.

A análise dos conteúdos foi realizada com o apoio do quadro operacional(11), utilizando as ferramentas de unidades de significância e reagrupamento temático. As apresentações descritivas, expondo as características metodológicas e os resultados, e sintéticas, resumindo as informações através dos quadros-síntese, visaram demonstrar o entendimento global dos estudos, sem, no entanto, negligenciar os aspectos específicos de cada uma das produções científicas investigadas.

\section{RESULTADOS}

\section{Estudos com Militares Federais}

Pereira e Teixeira ${ }^{(12)}$ realizaram uma pesquisa com militares da Aeronáutica $(n=1014)$ com o objetivo de estabelecer, por meio da avaliação e análise da aptidão física relacionada à saúde e a valores normativos para futuras avaliações, através de testes motores para verificar a resistência cardiorrespiratória, a flexibilidade e a resistência muscular localizada. Concluíram que os militares possuem níveis médios de desempenho físico, sendo que os homens apresentam um desempenho superior quando comparados às mulheres, com base em normas específicas por gênero. A qualidade física que mais se destaca nos militares é a resistência aeróbica. 
Maior e colaboradores ${ }^{(13)}$ desenvolveram pesquisas com o objetivo de quantificar a potência muscular de membros inferiores, bem como a composição corporal, antes e após o treinamento físico militar, três semanas com cinco sessões, de oficiais e sargentos do Centro de Instrução Pára-quedista General Penha Brasil - RJ $(n=10)$. Os autores deduziram que a influência da fadiga e do stress neuromuscular, associado ao reduzido consumo calórico, reduz a performance nos saltos verticais e nos horizontais, assim como produz alteração na composição corporal dos indivíduos participantes de um programa de treinamento físico militar.

Vieira e colaboradores ${ }^{(14)}$ efetuaram um estudo com o intuito de verificar os efeitos de oito semanas de Treinamento Físico Militar (TFM) no desempenho físico, freqüência cardíaca de repouso (FCR), pressão arterial sistólica (PAS) e somatório de dobras cutâneas, em integrantes de Forças de Paz $(n=30)$. O grupo foi divido em dois subgrupos, sendo um controle $(n=15)$ os quais não realizaram exercícios físicos e outro, de mesmo número de sujeitos ( $n=15)$, que efetuou sessões de trabalho cardiopulmonar e neuromuscular, ambos sendo submetidos a testes no início e no final das oito semanas. Os pesquisadores constataram diferenças significativas entre os grupos no que se refere ao somatório de no teste de corrida, porém não houve melhora significativa nos indicadores cardiovasculares.

Campos $^{(15)}$ comparou o efeito do aquecimento estático e dinâmico, bem como do alongamento, sobre o teste de força máxima em alunos do Instituto Militar de Engenharia (IME) $(n=30)$. Para tanto, demonstra que o Treinamento Físico Militar (TFM) realiza um aquecimento, composto de alongamento e exercícios de efeitos localizados que podem ser estáticos ou dinâmicos, e um trabalho principal que apresenta sessões neuromusculares (exercícios resistidos). Este autor concluiu que não houve diferença significativa no efeito provocado pelas diferentes formas de aquecimento sobre o teste de força máxima.

Santos e colaboradores ${ }^{(16)}$ realizaram um estudo com militares do $14^{\circ}$ Grupo de Artilharia de Campanha com o escopo de verificar como um programa de periodização do treinamento 16 semanas de trabalho de força e endurance, alteraria o resultado do teste de aptidão física (TAF) de 47 voluntários do sexo masculino. Dos sujeitos investigados, 28 apresentavam média de idade $(X)$ de 29,68 anos $(d p=6,55)$ e 19 apresentavam média de 28,68 anos $(X)(d p=5,85)$, sendo este últimos o Grupo Controle. Foram realizadas avaliações no inicio dos treinamentos e no final, verificando: medidas de peso, estatura, dobras cutâneas (subescapular, abdômen, supra-ilíaca, triciptal, biciptal, peitoral, médio-axilar, coxa e perna), circunferências (braço, antebraço, coxa, perna, todos direito e esquerdo, abdômen e tórax), diâmetro (bi-epicondiliano do úmero, bi-estilodial, bi-condiliano do fêmur e bimaleolar), além de testes de flexibilidade (teste de alcance máximo). Os pesquisadores identificaram diferenças significativas, somente, no grupo treinado, no consumo máximo de oxigênio, barra fixa e flexão de braços, concluindo que o treinamento periodizado em um grupo de militares resultou em melhoria nos indicativos de força para membros superiores (flexão de braço e barra fixa) e na capacidade aeróbia.

Miranda e colaboradores ${ }^{(17)}$ investigaram o efeito da marcha militar de $16 \mathrm{~km}$ sobre o índice de desidratação (Inddes), índices hematológicos, contagem de leucócitos e densidade de urina, segundo diferentes tipos de repositores hídricos. Compuseram este estudo 42 militares do Exército Brasileiro, do $1^{\circ}$ Esquadrão de Cavalaria Páraquedista, na cidade do Rio de Janeiro, sexo masculino, idade 22,6 $\pm 2,34$ anos, massa corporal $72,99 \pm 7,70 \mathrm{~kg}$ e peso transportado (material militar) $27,04 \pm 2,11 \mathrm{~kg}$. A amostra foi dividida, de forma aleatória, em quatro grupos, conforme o tipo de repositor utilizado (um litro por indivíduo): água mineral (Gp 1); sem re-hidratação (Gp 2); soro Babydrax (Gp 3); e Gatorade (Gp 4). Duas horas antes da marcha, foi realizada hidratação controlada de $600 \mathrm{ml}$ de água. Foram realizadas coleta de dados antes (A) e após (D) a marcha, das medidas de massa corporal (Pés) para avaliação da 
diferença percentual entre a massa pré e pós-marcha do Inddes, de densidade de urina (DensU), dos índices hematológicos (porcentagem do hematócrito$\mathrm{Hm}$ e contagem de hemoglobina- $\mathrm{Hb}$ ) e de contagem de leucócitos (Leuc). Os autores concluíram que a abstinência de líquidos, em atividade de marcha, pode levar à desidratação, e também devem ser levados em consideração situações climáticas e percursos maiores que podem ser percorridos. Caso não sejam utilizados os repositores a condição física dos envolvidos poderá estar prejudicada.

Rodrigues e colaboradores ${ }^{(18)}$ avaliaram o efeito do condicionamento físico aeróbico na resposta psicofisiológica a estressores laboratoriais em oficiais do Exército Brasileiro, submetendo 438 militares ao teste de 12 minutos de Cooper. Com base nos resultados o grupo foi subdividindo o grupo em dois subgrupos, um com os militares que percorreram mais de 3.200 metros e o outro com os que percorreram menos que 2400 metros, totalizando 28 sujeitos. Em seguida, estes foram submetidos a testes estressores laboratoriais (cold stressor e estressor matemático). Os autores verificaram que os indivíduos com melhor condição cardiorrespiratória tendem a apresentar padrões reduzidos na resposta autonômica ao estresse, como indicado pelo comportamento dos níveis de condutibilidade na pele.

Martins e Fernandes Filho(19) verificaram a validade concorrente de seis equações de predição generalizadas para a estimativa da densidade corporal em mulheres militares, mediante uma amostra composta por 107 mulheres militares, com idade entre 18 e 45 anos $(30,48 \pm 6,40$ anos $)$, massa corporal de $58,60 \pm 6,99 \mathrm{~kg}$, estatura de 164,78 $\pm 5,81 \mathrm{~cm}$, sendo mensuradas seis dobras cutâneas e uma circunferência conforme as equações selecionadas, concluindo que a equação quadrática generalizada de Petroski apud Martins e Fernandes Filho(19) $\mathrm{com}$ cinco dobras (subescapular, tríceps, supra-ilíaca, abdominal e panturrilha medial) possui validade concorrente para estimar a densidade corporal de mulheres militares.
Oliveira e Dos Anjos ${ }^{(20)}$ desenvolveram um trabalho com militares do sexo masculino, com o escopo de analisar as diferenças no índice de massa corporal (IMC), perímetro da cintura e aptidão cardiorrespiratória, mediante a antropometria (massa corporal, estatura e perímetro da cintura) e o consumo máximo de oxigênio (VO2max), estimado por meio do teste de corrida de 12 minutos. Os autores concluíram que os sujeitos com melhor condicionamento físico apresentam menor valor no perímetro da cintura e um acúmulo reduzido de gordura abdominal.

Dias e colaboradores ${ }^{(21)}$ buscaram verificar a influência do nível de condicionamento aeróbico sobre o rendimento na execução de uma pista de obstáculos e a realização de um teste de tiro em integrantes do Exército Brasileiro, fazendo parte deste estudo 28 militares homens, de 19 a 20 anos de idade. Foram utilizados os seguintes instrumentos e equipamentos: teste de Léger-Boucher, com o intuito de estimar a potência aeróbica máxima (VO2max); teste de tiro; pista de obstáculos. Os autores constataram que o nível de condicionamento aeróbico afeta o tempo de execução de uma pista de obstáculos e que existe relação entre o nível de condicionamento aeróbico e a diferença entre o resultado do tiro pós e pré-esforço como índice de performance no tiro (IPT).

Duarte e colaboradores ${ }^{(22)}$ investigaram o efeito de diferentes níveis de controle autonômico cardiovascular, medido por meio da potência de alta freqüência (HF) da Variabilidade da Freqüência Cardíaca (VFC), sobre a resposta de recuperação da Freqüência Cardíaca pós-exercício máximo, de indivíduos com mesma condição aeróbica. Os autores verificaram que a elevada potência de HF, reflete um maior controle vagal cardíaco, podendo acelerar a Freqüência Cardíaca de Recuperação pós-esforço máximo em homens jovens saudáveis.

Deste modo, considerando a necessidade de se proporcionar uma visão global dos estudos sobre militares federais analisados nesta pesquisa, é possível apresentar o QUADRO-SÍNTESE 1, através da FIGURA 2. 
FIGURA 2

QUADRO-SÍNTESE DE ARTIGOS CIENTÍFICOS SOBRE MILITARES FEDERAIS

\begin{tabular}{|c|c|c|}
\hline \multirow{2}{*}{ ARTIGOS } & \multicolumn{2}{|r|}{ SÍNTESES } \\
\hline & Sujeitos & Temáticas dos Estudos \\
\hline Pereira e Teixeira ${ }^{(12)}$ & $\begin{array}{l}\text { Militares da } \\
\text { Aeronáutica, Homens e } \\
\text { Mulheres, } n=1014 .\end{array}$ & $\begin{array}{l}\text { - Resistência cardiorrespiratória; } \\
\text { - Flexibilidade; } \\
\text { - Resistência muscular localizada. }\end{array}$ \\
\hline Maior e Cols ${ }^{(13)}$ & $\begin{array}{l}\text { Militares do Exército, } \\
\text { Homens, } n=10 \text {. }\end{array}$ & $\begin{array}{l}\text { - Potência muscular de membros inf; } \\
\text { - Composição corporal; } \\
\text { - Treinamento físico Militar. }\end{array}$ \\
\hline Vieira e Cols ${ }^{(14)}$ & $\begin{array}{l}\text { Militares, integrantes de } \\
\text { forças de paz, Homens, } \\
\qquad n=30 .\end{array}$ & $\begin{array}{l}\text { - Treinamento Físico Militar; } \\
\text { - Freqüência Cardíaca de Repouso; } \\
\text { - Pressão Arterial Sistólica; } \\
\text { - Somatório de dobras cutâneas }\end{array}$ \\
\hline Campos e Cols ${ }^{(15)}$ & $\begin{array}{l}\text { Alunos do Instituto } \\
\text { Militar de Engenharia, } \\
\text { Homens, } n=30 \text {. }\end{array}$ & $\begin{array}{l}\text { - Treinamento Físico Militar; } \\
\text { - Aquecimento estático e dinâmico; } \\
\text { - Alongamento; } \\
\text { - Força máxima. }\end{array}$ \\
\hline $\begin{array}{c}\text { Santos, Paiva Neto } \\
\text { e Peres }\end{array}$ & $\begin{array}{l}\text { Militares do Exército, } \\
\text { Homens, } n=47 \text {. }\end{array}$ & $\begin{array}{l}\text { - Periodização de Treinamento; } \\
\text { - Treinamento de Força; } \\
\text { - Treinamento de Endurance; } \\
\text { - Teste de Avaliação Física (TAF). }\end{array}$ \\
\hline Miranda e Cols ${ }^{(17)}$ & $\begin{array}{l}\text { Militares do Exército, } \\
\text { Homens, } n=42 \text {. }\end{array}$ & $\begin{array}{l}\text { - Marcha Militar; } \\
\text { - Índice de Desidratação (Inddes); } \\
\text { - Repositores hídricos; }\end{array}$ \\
\hline Rodrigues e Cols ${ }^{(18)}$ & $\begin{array}{l}\text { Oficiais Militares do } \\
\text { Exército, } \\
\text { Homens, } n=438 \text {. }\end{array}$ & $\begin{array}{l}\text { - Condicionamento físico aeróbio; } \\
\text { - Respostas psicofisiológicas. }\end{array}$ \\
\hline $\begin{array}{c}\text { Martins e Fernandes } \\
\text { Filho }^{(19)}\end{array}$ & $\begin{array}{l}\text { Militares do Exército, } \\
\text { Mulheres, } 18 \text { a } 45 \\
\text { anos, } n=107 .\end{array}$ & $\begin{array}{l}\text { - Equações para estimativa de } \\
\text { densidade corporal; }\end{array}$ \\
\hline $\begin{array}{l}\text { Oliveira e Dos } \\
\text { Anjos }^{(20)}\end{array}$ & $\begin{array}{l}\text { Militares do Exército, } \\
\text { Homens, } n=50.523 \text {. }\end{array}$ & $\begin{array}{l}\text { - índice de Massa Corporal; } \\
\text { - Perímetro da cintura; } \\
\text { - Aptidão cardiorrespiratória. }\end{array}$ \\
\hline Dias e Cols ${ }^{(21)}$ & $\begin{array}{l}\text { Militares do Exército, } \\
\text { Homens, } 19 \text { a } 20 \text { anos, } \\
\text { n= } 28 .\end{array}$ & $\begin{array}{l}\text { - Condicionamento aeróbio; } \\
\text { - Pista de obstáculos; }\end{array}$ \\
\hline Duarte e Cols ${ }^{(22)}$ & $\begin{array}{l}\text { Militares do Exército, } \\
\text { Homens, } 18 \text { a } 25 \text { anos, } \\
\text { n= } 100 .\end{array}$ & $\begin{array}{l}\text { - Controle autonômico } \\
\text { cardiovascular; } \\
\text { - Potência de alta FC; } \\
\text { - Variabilidade da FC; } \\
\text { - Recuperação da FC }\end{array}$ \\
\hline
\end{tabular}

\section{Estudos com Militares Estaduais}

Domingues $^{(23)}$ realizou um estudo com Policiais Militares (PMs) do estado de Mato Grosso identificando que $47,35 \%$ dos entrevistados praticam alguma atividade física e $27,35 \%$ afirmaram não praticar atividade física alguma no decorrer da semana. Destes
$85 \%$ realizam atividade física sem acompanhamento de profissional de Educação Física ou mesmo da saúde, tornando a atividade perigosa para seus praticantes.

Bezerra Filha ${ }^{(24)}$ efetuou um trabalho com setenta PMs do estado da Paraíba, do sexo masculino, em João Pessoa com média de idade de 33 anos. Informa que devido ao fato de atuarem em condições desfavoráveis, atendendo situações de emergência e de risco sua capacidade física deve estar em pleno desenvolvimento. Sua pesquisa apresentou um resultado não adequado para a necessidade do serviço, ou seja, $66 \%$ dos avaliados apresentavam sobrepeso, $53 \%$ apresentaram risco elevado de desenvolver doenças cardiovasculares e $74 \%$ apresentaram níveis insuficientes em pelo um dos componentes da aptidão física relacionada à saúde.

Rodriguez-Añez ${ }^{(25)}$ desenvolveu uma pesquisa com 369 PMs do Estado de Santa Catarina, todos do sexo masculino e que ingressaram na corporação em 2002 com média de idade de 22,7 anos, com o intuito de elaborar um sistema de avaliação para a promoção e gestão do estilo de vida saudável e da aptidão física relacionada à saúde de PMs. Para tanto, foi utilizado o questionário Estilo de Vida Fantástico e a bateria de testes Plano de Avaliação da Atividade Física, Aptidão e Estilo de Vida, propostos pela Sociedade Canadense para a Fisiologia do Exercício para avaliação da aptidão físicam. A aptidão cardiorrespiratória foi avaliada por meio do teste de Vai-e-Vem de Léger. O autor concluiu que o grupo se caracteriza com uma boa condição para a saúde, porém alguns dados devem ser levados em consideração, pois $72,9 \%$ não praticam atividade física de vigorosa intensidade na freqüência recomendada, $56,1 \%$ não praticam atividade física moderada, $33,9 \%$ não seguem uma dieta balanceada, $9,8 \%$ fumam e $11,1 \%$ bebem.

Boldori e colaboradores ${ }^{(26)}$ realizaram um estudo com 359 Bombeiros Militares (BMs) do estado de Santa Catarina com o objetivo de investigar a aptidão 
física e a relação com a capacidade de trabalho. Foi utilizado teste de Léger (avaliar a potência aeróbica máxima), força dos membros superiores, exercício de suspensão na barra fixa, resistência abdominal, velocidade máxima nos 50 metros e composição corporal. Para verificar a capacidade de trabalho foi utilizado o Índice de Capacidade para o Trabalho (ICT). A média do percentual de gordura foi de $15,0 \%$, sendo que $5,29 \%$ dos avaliados estão classificados como obesos, $27,29 \%$ como sobrepesos e $67,30 \%$ como bom, muito bom e excelente. A aptidão física indicou que $11,7 \%$ dos avaliados têm sua aptidão física considerada insuficiente e 88,3\% regular, bom, muito bom e excelente. Os autores concluíram que o nível de aptidão física e o percentual de gordura são inversamente proporcionais à idade e existe correlação entre a Aptidão Física e a Capacidade de Trabalho dos Bombeiros Militares do Estado de Santa Catarina.

Mattos e colaboradores ${ }^{(27)}$ efetuaram um estudo com Bombeiros Militares Comunicantes (BMC) $(n=66)$ do Estado do Rio de Janeiro, com o escopo de avaliar a influência do antecedente familiar de hipertensão arterial sistêmica (HASF) sobre o efeito do estresse do trabalho, através da monitorização ambulatorial da pressão arterial (MAPA). A pressão arterial mais elevada em BMC filhos de hipertensos é explicada de maneira independente pela HASF e aqueles que desenvolveram hipertensão arterial sistêmica (HAS) durante o turno de trabalho, podem ser considerados hipertensos funcionais, enquanto, os BMC filhos de normotensos, submetidos ao estresse psicológico, estão livres de alterações na pressão arterial.

Silva(28), desenvolveu um estudo com Policiais Militares do estado do Mato Grosso, com o objetivo de identificar as fontes de estresse, bem como os níveis de estresse produzidos por estas fontes e sua influência na atividade operacional. O pesquisador identificou inúmeras fontes que somadas aos problemas internos, provocam reações que vão se acumulando no dia-a-dia desses homens podendo ocasionar seqüelas físicas e emocionais irreparáveis quando não diagnosticadas e tratadas a tempo.

Assim sendo, a partir da necessidade de se demonstrar uma visão global dos estudos sobre militares estaduais analisados nesta pesquisa bibliográfica, é possível apresentar o QUADROSÍNTESE 2, através da FIGURA 3.

\section{CONCLUSÕES}

A partir das bases de dados investigadas, foi possível constatar a existência de diferentes tipos de produção científica produzida no Brasil no período entre janeiro de 2003 e março de 2008 envolvendo o descritor "militar", pois abrange uma grande quantidade de profissionais federais (membros das forças armadas) e estaduais (policiais e bombeiros militares). Contudo, quando cruzado com os descritores secundários, em específico a "atividade física" e "qualidade de vida", pode-se verificar que a quantidade de estudos diminui significativamente, principalmente quanto aos militares estaduais.

Considerando a produção científica sobre militares federais, pode-se concluir que os aspectos que apresentam maior relação com os descritores secundários são aqueles principalmente relacionados ao treinamento militar, tais como resultados aeróbicos e anaeróbicos, respostas do sistema cardiovasculares, respostas psíquicas, respostas do sistema osteomioarticular, respostas do sistema metabólico, respostas ao aquecimento estático e dinâmico, treinamento de resistência e força muscular, tipo de treinamento e Treinamento Físico Militar (TAF). Já os aspectos com menor relação com os descritores secundários, referem-se à aptidão física relacionada à saúde, tais como alongamento e flexibilidade, força e resistência muscular, aptidão física, composição corporal.

Considerando a produção científica sobre militares estaduais, pode-se concluir que os aspectos que apresentam maior relação com os descritores secundários são aqueles principalmente 
FIGURA 3

QUADRO-SÍNTESE DE ARTIGOS CIENTÍFICOS SOBRE MILITARES ESTADUAIS

\begin{tabular}{|c|c|c|}
\hline \multirow{2}{*}{ ARTIGOS } & \multicolumn{2}{|c|}{ SÍNTESE } \\
\hline & Sujeitos & $\begin{array}{l}\text { Temáticas dos } \\
\text { Estudos }\end{array}$ \\
\hline Domingues $^{(23)}$ & $\begin{array}{c}\text { Policiais Militares } \\
\text { (MT), Homens, } \\
\text { n= } 118\end{array}$ & $\begin{array}{c}\text { - Nível de atividade } \\
\text { física; }\end{array}$ \\
\hline Bezerra Filha ${ }^{(24)}$ & $\begin{array}{c}\text { Policiais Militares } \\
\text { (PB), Homens, } \\
n=70\end{array}$ & $\begin{array}{c}\text { - Índice de Massa } \\
\text { Corporal; } \\
\text { - Aptidão Física } \\
\text { relacionada à Saúde; } \\
\text { - Risco cardiovascular. }\end{array}$ \\
\hline Rodriguez-Añez ${ }^{(25)}$ & $\begin{array}{c}\text { Policiais Militares } \\
\text { (SC), Homens, } \\
n=369\end{array}$ & $\begin{array}{l}\text { - Avaliação Física; } \\
\text { - Estilo de Vida; } \\
\text { - Aptidão } \\
\text { cardiorrespiratória. }\end{array}$ \\
\hline Boldori e Cols ${ }^{(27)}$ & $\begin{array}{c}\text { Bombeiros } \\
\text { Militares (SC), } \\
\text { Homens, } n=359\end{array}$ & $\begin{array}{l}\text { - Aptidão física; } \\
\text { - Capacidade de } \\
\text { trabalho. }\end{array}$ \\
\hline Mattos e Cols ${ }^{(28)}$ & $\begin{array}{c}\text { Bombeiros } \\
\text { Militares (RJ), } \\
\text { Homens, } n=66\end{array}$ & $\begin{array}{c}\text { - Antecedência de } \\
\text { hipertensão arterial } \\
\text { sistêmica; } \\
\text { - Estresse no trabalho; } \\
\text { - Pressão Arterial } \\
\text { Sistêmica; } \\
\text { - Hipertensão arterial. }\end{array}$ \\
\hline Silva ${ }^{(29)}$ & $\begin{array}{c}\text { Policiais Militares } \\
\text { (MT), Homens, } \\
n=45\end{array}$ & $\begin{array}{l}\text { - Fontes de estresse; } \\
\text { - Níveis de estresse; } \\
\text { - Atividade profissional. }\end{array}$ \\
\hline
\end{tabular}

relacionados à aptidão física relacionada à saúde, tais como a aptidão física, estresse; tensão arterial, composição corporal, nível de atividade física, risco cardiovascular. Já os aspectos com menor relação com os descritores secundários, referem-se à atividade profissional, capacidade de trabalho e o estilo de vida.

Portanto, sugere-se que o número de estudos com militares apresente um incremento, principalmente com os estaduais, pois ainda se verifica carência de estudos científicos disponíveis na bibliografia brasileira.

\section{REFERÊNCIAS BIBLIOGRÁFICAS}

1. Silva R. Características do estilo de vida e da qualidade de vida de professores do ensino superior público em educação física. Tese apresentada ao Programa de PósGraduação em Engenharia de Produção, da Universidade Federal de Santa Catarina. 2006.

2. Hossri CA. O paradoxo do exercício: doses adequadas e benefícios, doses inadequadas e riscos. Rev Educ Fís 2007;137:70-3.

3. Forattini OP. A saúde pública no século XX. Rev Saúde Pública 2000;211-13.

4. Costa M, Accioly Júnior H, Oliveira J, Maia E. Estresse: diagnóstico dos policiais militares em uma cidade brasileira. Rev Panam Salud Publica 2007;21(4):217-22.

5. Lida I. Ergonomia: Projeto e Produções. São Paulo: Edgar Blücher, 1992.

Dejours C. A loucura do trabalho. São Paulo: Oboré, 1987.

6. Forattini OP. Ecologia, epidemiologia e sociedade. São Paulo: Artes Médicas, 1992.

7. Brasil. Constituição do. Brasília: Senado Federal, Centro Gráfico, 1988.

8. Brasil. Decreto-Lei $n^{\circ}$ 667, de 2 de julho de 1969. Reorganiza as Polícias Militares e os Corpos de Bombeiros Militares dos Estados, do Território e do Distrito Federal, e dá outras providências.

9. Santos Júnior AA, Dutra LH, Silva Filho DB. Levantamento da percepção do medo e do crime em Santa Catarina. Revista Brasileira de Segurança Pública 2007;1(2):94-119.

10. Alves-Mazzotti AJ, Gewandsznajder F. O método nas ciências naturais e sociais: Pesquisa quantitativa e qualitativa. 2 ed. São Paulo: Pioneira Thomson Learning, 2002.

11. Laville C, Dione J. A construção do saber: Manual de metodologia da pesquisa em ciências humanas. Porto Alegre: Artes Médicas; Belo Horizonte: EUFMG, 1999.

12. Pereira ÉF, Teixeira CS. Proposta de valores normativos para avaliação da aptidão física em militares da Aeronáutica. Rev Bras Educ Fis Esp 2006;20(4):249-56.

13. Maior AS, Souza MWBJ, Defilippo E, Granado FD, Boabaid JWS, Beyruth RMP, et al. Efeitos do treinamento físico militar na potência muscular dos membros inferiores e nos indicadores da composição corporal. Rev Educ Fís 2006;135:5-12. 
14. Vieira G, Duarte D, Silva R, Fraga C, Oliveira M, Rocha $\mathrm{R}$, et al. Efeitos de oito semanas de treinamento físico militar sobre o desempenho físico, variáveis cardiovasculares e somatório de dobras cutâneas de militares de força de paz do exército brasileiro. Rev Educ Fís 2006;20(134):30-40.

15. Campos GKD, Mendes FVC, Alves Júnior JMM, Brandão ALC, Santos, ES, Souza LAC, et al. O efeito de diferentes formas de aquecimento sobre teste de força máxima em alunos do instituto militar de engenharia. Rev Educ Fís 2006;135:28-35.

16. Santos A, Paiva Neto A, Peres FP. Influência do treinamento combinado de força e endurance nas respostas do TAF de militares do $14^{\circ} \mathrm{GAC}$. Movimento e Percepção 2005;5(6):163-75.

17. Miranda GSM, Ramos CEG, Vasco LG, Reis LVM, Lemos BR, Sovat, VA, et al. Efeito da marcha militar de $16 \mathrm{Km}$ em índice de desidratação, índices hematológicos, contagem de leucócitos e densidade de urina, segundo a reposição hídrica. Rev Educ Fís 2007;138(4):4-12.

18. Rodrigues AVS, Martinez EC, Duarte AFA, Ribeiro LCS. $O$ condicionamento aeróbio e sua influência na resposta ao estresse mental em oficiais do Exército. Rev Bras Med Esporte 2007;13(2):113-18.

19. Martins MEA, Fernandes Filho J. Validação de equações antropométricas generalizadas para a estimativa da densidade corporal em mulheres militares. Rev Bras Cineantropom Desempenho Hum 2006;8(1):22-8.

20. Oliveira EAM, Dos Anjos LA. Medidas antropométricas segundo aptidão cardiorrespiratória em militares da ativa, Brasil. Rev Saúde Pública 2007;42(2):217-23.

21. Dias AC, Dantas EHM, Moreira SB, Silva VF. A relação entre o nível de condicionamento aeróbico, execução de uma pista de obstáculos e o rendimento em um teste de tiro. Rev Bras Med Esporte 2005;11(6):341-46.

22. Duarte A, Andrade Neto AM, Abreu DC, Pereira EM, Modesto GE, Martins JCL, et al, A influência do controle autonômico na recuperação da freqüência cardíaca pósesforço de militares do Exército Brasileiro. Rev Educ Fís 2007;139:13-9.
23. Domingues EMM. Saúde Policial e Qualidade de Vida. Monografia de Especialização em Gestão de Segurança Pública apresentada ao Programa de Pós-Graduação em Segurança Pública, da Universidade Federal de Mato Grosso. 2003.

24. Bezerra Filha MJA. Níveis de aptidão física relacionados à saúde dos policiais militares que trabalham no serviço de rádio-patrulha do $5^{\circ}$ Batalhão de João Pessoa. Monografia de Especialização em Gestão de Segurança Pública apresentada ao Programa de Pós-Graduação em Segurança Pública, da Academia de Polícia Militar da Paraíba. 2004.

25. Rodriguez-Añez CR. Sistema de avaliação para a promoção e gestão do estilo de vida saudável e da aptidão física relacionada à saúde de policiais militares. Tese apresentada ao Programa de Pós-Graduação em Engenharia de Produção, da Universidade Federal de Santa Catarina. 2003.

26. Boldori R, Petroski EL, Silveira JLG, RodriguezAñez CR. Aptidão física, saúde e índice de capacidade de trabalho de bombeiros. Lecturas: Educación Física y Deportes [on-line]. 2005; 80. Disponível em: www. efdeportes.com/efd80/bombeiro.htm (10 mar. 2008).

27. Mattos CE, Mattos MA, Toledo DC, Siqueira Filho AG. Avaliação da pressão arterial em bombeiros militares filhos de hipertensos através da monitorização ambulatorial da pressão arterial. Arq Bras Cardiol 2006;86(6):741-46.

28. Silva D. Estresse policial no $3^{\circ}$ BPM da cidade de Cuiabá-MT. Monografia de Especialização em Gestão de Segurança Pública apresentada ao Programa de Pós-Graduação em Segurança Pública, da Universidade Federal de Mato Grosso. 2003.

\section{Endereço para correspondência:}

Rua Paschoal Simone, N 358, Bairro Coqueiros - Florianópolis - SC - Brasil CEP: 88080-350.

Telefone: (48) 3321-8651 Fax: (48) 3321-8607. E-mail: melo2@pm.sc.gov.br, rudney@udesc.br. 